\title{
Research on Multi-robot Cooperation Based on Leader-follower Method
}

\author{
Yingjie Huang $^{1}$, Yong Zhang ${ }^{2}$, Qinjun Zhao ${ }^{3}$ and Jin Cheng ${ }^{4}$ \\ School of Electrical Engineering,University of Jinan,Jinan, China \\ e-mail: hyjde163@163.com ${ }^{1}$, cse_zhangy@ujn.edu.cn ${ }^{2}$, cse_zhaoqj@ujn.edu.cn $^{3}$, \\ cse_chengj@ujn.edu.cn ${ }^{4}$
}

Keywords:Multi-robots; cooperative transportation; Leader-follower

\begin{abstract}
Compared with single robot system, multi-robot system has many outstanding advantages, such as parallelism, redundancy and spatial distribution characteristics. And at present, multi-robots system is already successfully applied in aerospace, automotive assembly welding, disaster relief and other fields. In this paper we specify the implementation of two mobile robots carrying a large size box to a given destination cooperatively, which is a typical example of multirobot system. Firstly, inspired by the formation control, the leader-follower mechanism, which is often applied in the formation control, is introduced in this paper. Secondly, based on $l-\phi$ control mode of the leader-follower mechanism, a PID controller, whose input deviation is calculated by LOS (line-of-sight) method, for the leader robot, while a double input dual output fuzzy controller are designed for the follower robot to complete cooperative transport task. Lastly, an application written in $\mathrm{C}++$ language is developed to verify the proposal. The experiment that controlling two amigo bots to transport a big size box to a given destination cooperatively is carried out in the indoor environment, and the experimental results show that the proposed method is effective.
\end{abstract}

\section{Introduction}

With the development of society and technology, the requirements for robots are no longer just simple repetitive movements, but also some intelligent synergy. Some of these requirements for single robots are highly cost while others are beyond their capabilities. Especially when there is a complex division of labor and cooperation of operational requirements, multiple robots is needed to complete the task by collaboration. Therefore, more and more scholars began to study the multirobot system $[1,2]$.

The scene that two people carrying an object cooperatively can often be seen in life. There is also such a job requirement in the industrial field [3]. Therefore, this paper studies how to implement two amigo bots, designed by adept company, to carry a large size box to a specified destination cooperatively. Since the box is rectangular and its size is much larger than a single amigo bot, it is difficult for a single amigo bot to complete the task. Therefore, this paper introduces multiple mobile robots to complete the task cooperatively. The key for cooperative task is that the robots should be able to adjust their position relationship and the direction of motion to generate a cooperative force, so they can eventually push the box to the given destination. The diagram is shown in figure 1 .

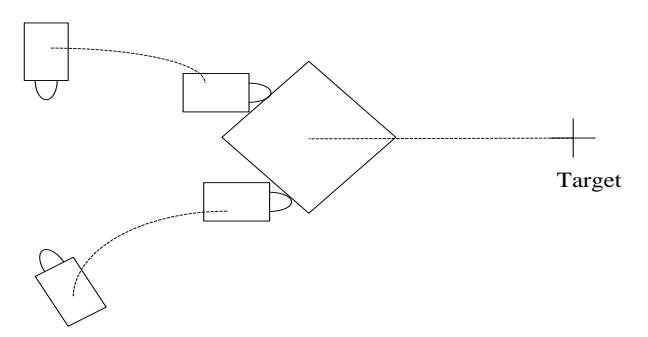

Figure 1. Schematic diagram of collaborative transport 


\section{a proposal for collaborative transport}

The essence of collaborative transport is the control of both the positional relationship and direction of movement of the robots, which is similar to formation control. Currently there are three commonly used formation control methods: behavior-based approach[4], the virtual structure method[5] and the leader-follower formation control method[6, 7].

In the multi-robot system, one robot is selected firstly as a system leader and the other robots as followers. The main idea of leader-follower method is to transform the formation control problem into keeping a certain distance and angular difference between the followers and the leader. Then a controller is designed to make the followers to keep a certain distance and angle with the leader respectively. There are three kinds of controller in the Leader-Follower control mode shown as follows:

\section{A. $l-\phi$ controller}

Set the distances and relative angle values between the leader and followers to be constants. In the formation control of robots, followers always monitor their selected leader, through some kind of control algorithms to keep their distance and azimuth converge as the desired value.

\section{B. $l-l$ Controller}

Set the relative distances between robots to be constant. There are certain distances between followers and leader, and the convergence of the distance should be kept in a deviation range.

\section{Mixed control}

It refers to the combination of both ${ }^{l-\phi}$ and ${ }^{l-l}$ control methods, it has advantages of both methods.

In this paper, the ${ }^{l-\phi}$ control method is enough for us.

\section{Leader controller}

In leader-follower mechanism the leader is supposed to be not affected by the follower robot, so in this paper, the leader only needs to track to the straight line which will lead the box reach the target. Therefore, the leader controller is actually a path tracking controller and we select LOS PID controller here.

\section{A. LOS method}

The position relationship between the robot and the target path, including the distance and angle information, should be clear when the robot is tracking a straight path. The LOS angle calculated by LOS (Line-Of-Sight) method[8], contains not only distance information but also angle information need by the path tracking. The LOS method was shown in figure 2 .

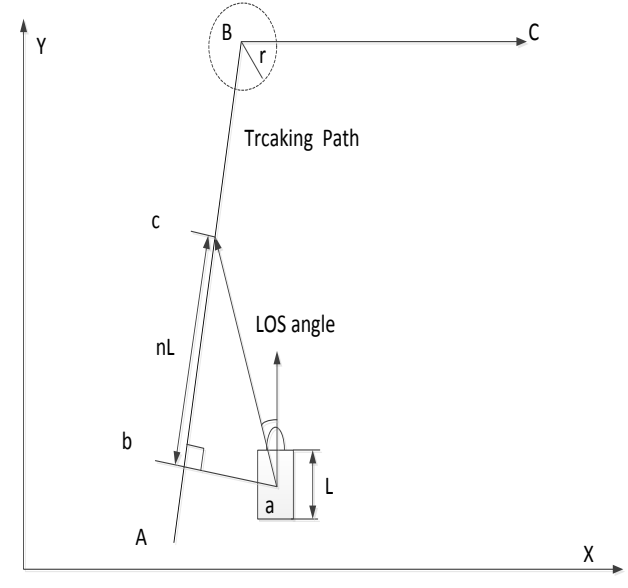

Figure 2. Schematic diagram of LOS method 
- Assuming that the tracking path is from point $\mathrm{A}$ to point $\mathrm{B}$ to point $\mathrm{C}$, and the current tracking straight line is $\mathrm{AB}$, then each line segment can be determined by two points

- Make a vertical line from the point which is the central position of the robot to the line AB. The pedal point is named as $b$.

- Draw a line segment bc whose length is $\mathrm{nL}$ on line $\mathrm{AB}$. ( $\mathrm{n}$ is a constant number, $\mathrm{L}$ is the length of the body of the robot)

- Connect point a with point $\mathrm{c}$ and the direction from a to $\mathrm{c}$ will be the heading of the robot in the next movement

- The angle difference between the current heading of the robot and the ac direction is called LOS angle;

- When the distance from the robot to the corner of the tracking path is less than $\mathrm{r}$, the current tracking straight line changes from $\mathrm{AB}$ to $\mathrm{BC}$, which enables the robot to track the full target path.

\section{B.PID controller}

LOS PID controller refers to a PID controller, which is designed to achieve the path tracking. The input offset of the controller is LOS angle calculated by LOS method. In this paper we also make the speed difference of robot's left and right wheel as the controller's output variable. Controller system diagram is shown in figure 3.

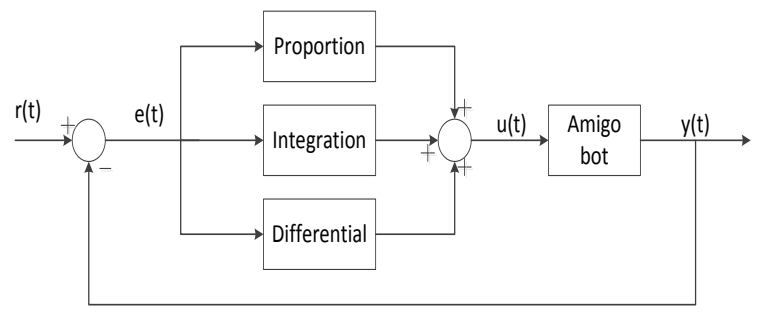

Figure 3. Schematic diagram of LOS-PID Controller

The PID control law is:

$$
u(t)=k_{p}\left(e(t)+\frac{1}{T_{l}} \int e(t) d t+\frac{T_{d} e(t)}{d t}\right)
$$

And the transfer function form is:

$$
G(s)=\frac{U(s)}{E(s)}=k_{p}\left(1+\frac{1}{T_{l} S}+T_{d} S\right)
$$

The ${ }^{k_{p}}$ represents the ratio of the coefficient, ${ }^{T_{l}}$ indicates the integration time and ${ }^{T_{d}}$ said the derivative time constant. The test method was used to determine the specific values for each parameter here

\section{Follower controller}

The ${ }^{l-\varphi}$ control mode[9] is shown in figure 4. $V_{L}$ is the velocity vector of the leader robot. $V_{F}$ is the velocity vector of the follower robot. $\mathrm{P}$ is the expected position or tracking point for the follower robot. The distance between the point $\mathrm{P}$ and the leader robot is ${ }^{l} . \varphi$ is the angle from the connection of point $\mathrm{P}$ and the leader robot to the horizontal line. ${ }^{\theta}$ is the angle from the connection of point $\mathrm{P}$ and the follower robot to the $V_{F}$ vector. The dist is the distance between the follower robot and the tracking point $\mathrm{P}$ 。 


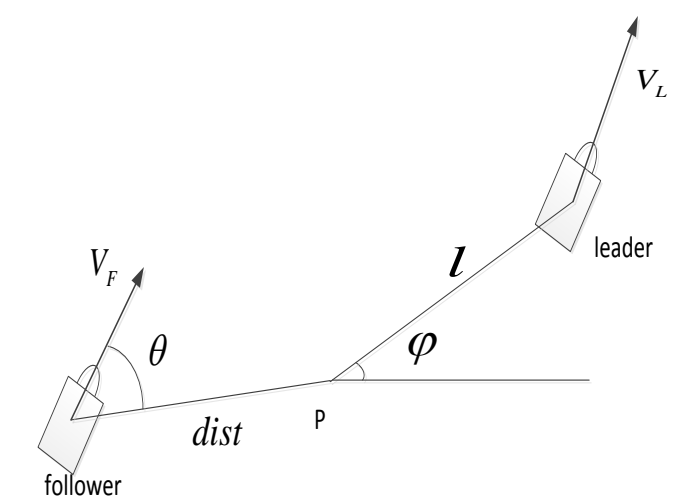

Figure 4. Schematic diagram of $l-\phi$ control mode

In ${ }^{l-\varphi}$ control mode, the follower robot should keep a constant distance ${ }^{l}$ and angle ${ }^{\varphi}$ with the leader robot, which requires the follower robot to track point $P$. we will use a double-input doubleoutput fuzzy controller to achieve the requirement in this paper[10, 11]. The first input variable of the controller is the distance between the follower and the desired position $\mathrm{P}$, and the second input is the angle deviation $\theta$ between $V_{F}$ and the connected line from the follower to the desired point $\mathrm{P}$. The first output of the controller is to determine the direction of the follower robot's velocity vector $V_{F}$, and the second output of the controller refers to the amplitude of the follower robot's velocity vector $V_{F}$.

The velocity of the amigo bot's left and right wheels is calculated as follows

$$
\left\{\begin{array}{l}
V_{l}=V_{c}-E_{v} / 2 \\
V_{r}=V_{c}+E_{v} / 2
\end{array}\right.
$$

In above formula $V_{r}$ refers to the velocity of the right wheel, $V_{l}$ means to the velocity of the left wheel and $V_{c}$ is the basic speed。

The direction of the velocity vector $V_{F}$ is determined by the $E_{v}$ variable. When $E_{v}$ is a negative number, the robot will turn to right. When $E_{v}$ is a positive number, it means the robot will turn to left. And the majority of the velocity vector $V_{F}$,s amplitude is depended on the basic speed $V_{c}$.

We define fuzzy linguistic variables sets in their basic domain for input variables dist $\theta$ and output variables $E_{v}, V_{c}$ respectively, as follows:

- $\quad E d=F($ dist $)=\{Z, N, F\}$, refers that the distance from the desired position $P$ to the follower robot is zero, near and far respectively.

- $\mathrm{Ea}=\mathrm{F}(\theta)=\{\mathrm{NB}$, NS, ZE, PS, PB $\}$, the angle from the connection between the follower robot and the desired point $\mathrm{P}$ is negative large, negative small, zero, positive small, positive large. And the basic domain was defined as $(-180,180]$.

- $\mathrm{Ud}=\mathrm{F}\left(E_{v}\right)=\{\mathrm{TLB}, \mathrm{TLM}, \mathrm{TLS}, \mathrm{ZE}, \mathrm{TRS}, \mathrm{TRM}, \mathrm{TRB}\}$. Respectively, they mean that in the next moment the robot will turn left big, turn left medium, and turn left small, straight ahead, turn right small, turn right medium, turn right big, in the next moment. And we take the basic domain as $[-1,1]$.

- $\mathrm{Uv}=\mathrm{F}\left(V_{c}\right)=\{\mathrm{SZ}, \mathrm{SL}, \mathrm{SH}\}$. Respectively, they indicate that the speed rate of the follower robot is zero, low and high. The basic domain was taken as $[0,1]$.

The membership functions of each member in fuzzy variable set Ed, Ea, Ud, Uv are shown respectively in figure 5 , figure 6 , figure 7 and figure 8. 


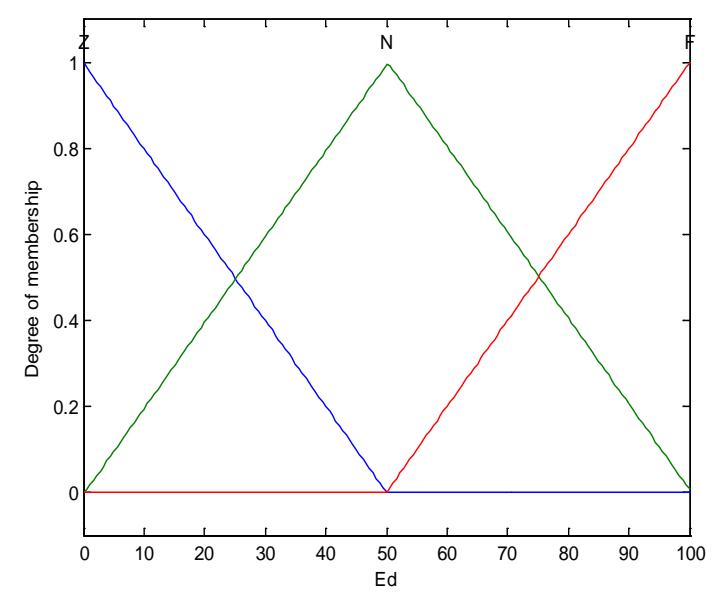

Figure 5. The membership functions of the follower controller input Ed

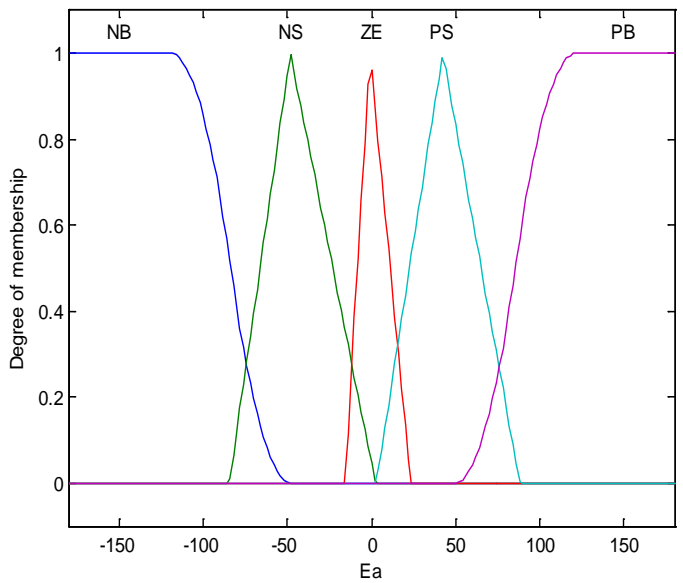

Figure 6. The membership functions of the follower controller input Ea

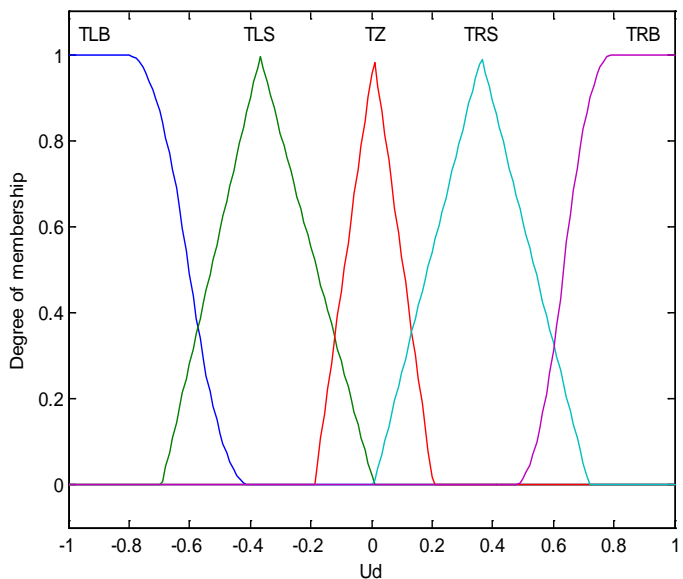

Figure 7. The membership functions of the follower controller output Ud 


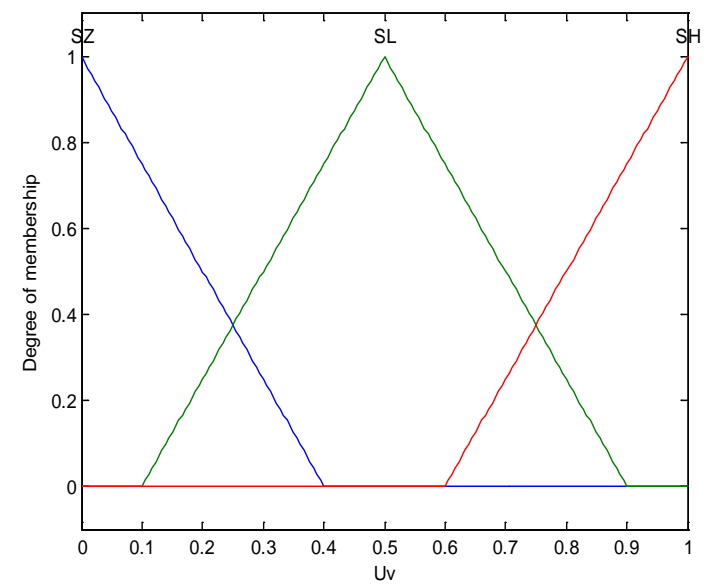

Figure 8. The membership functions of the follower controller output Uv

According to the positional relationship between point $\mathrm{P}$ and the follower, different rules are required to be designed to make the follower to track point $P$. The table of fuzzy rules is shown in Table 1.

TABLE I. THE TABLE OF FUZZY RULES

\begin{tabular}{|c|c|c|c|}
\hline $\mathrm{Ea}_{\mathrm{Ea}}^{\mathrm{Ed}}$ & $Z$ & $N$ & $F$ \\
\hline$N B$ & $T L B S Z$ & $T L B S L$ & $T L B S H$ \\
\hline$N S$ & TLS SZ & TLS SL & TLS SH \\
\hline$Z E$ & TZ SZ & TZ SL & $T Z S H$ \\
\hline$P S$ & TRS SZ & TRS SL & TRS SH \\
\hline$P B$ & $\begin{array}{l}\text { TRB } \\
\text { SZ }\end{array}$ & $T R B S L$ & TRB SH \\
\hline
\end{tabular}

\section{Experiment Result}

In this paper, the leader-follower mechanism was realized by an MFC application, which implemented two amigo bots to carry a box cooperatively to the specified destination. The effect of LOS PID controller was shown in figure 9, which said the distance between the leader to the tracking line was around zero, and the deviation was less than $140 \mathrm{~mm}$. The first fuzzy controller input variable Ed shown in figure 10 represented the distance between the desired position and the follower, which said the majority of the distance deviation was kept in $100 \mathrm{~mm}$, and always less than $200 \mathrm{~mm}$. In fact due to the movement of the leader was a stochastic process, the accuracy of tracking process was hardly to be remain as zero. Figure 11 was the trajectory of the box under the cooperative implement by the amigo bot, wherein the asterisk indicates the transporting target. We can saw in Figure 12 that the box was gradually moved to the target under the synergistic effect, and finally the deviation was around $50 \mathrm{~mm}$. 


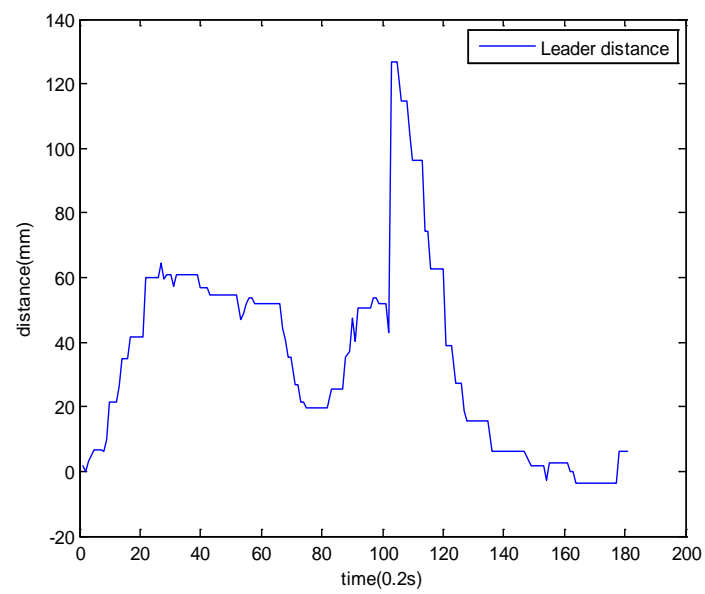

Figure 9. Distance between the leader and the tracking path

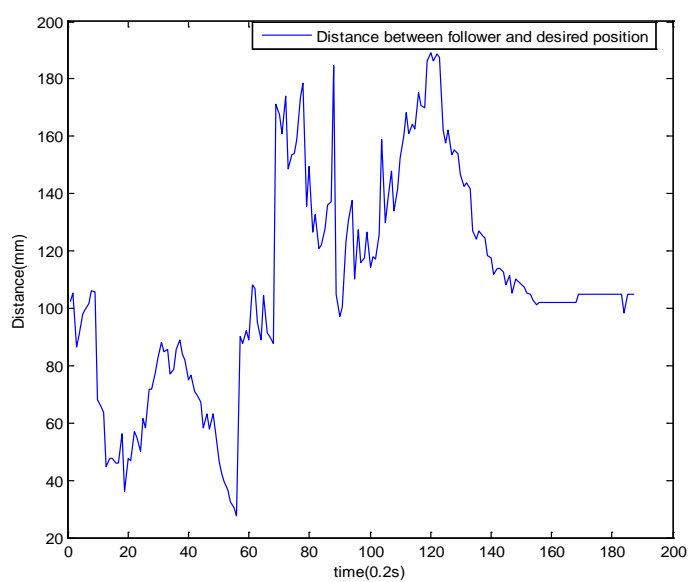

Figure 10. The distance deviation from the follower to the desired position

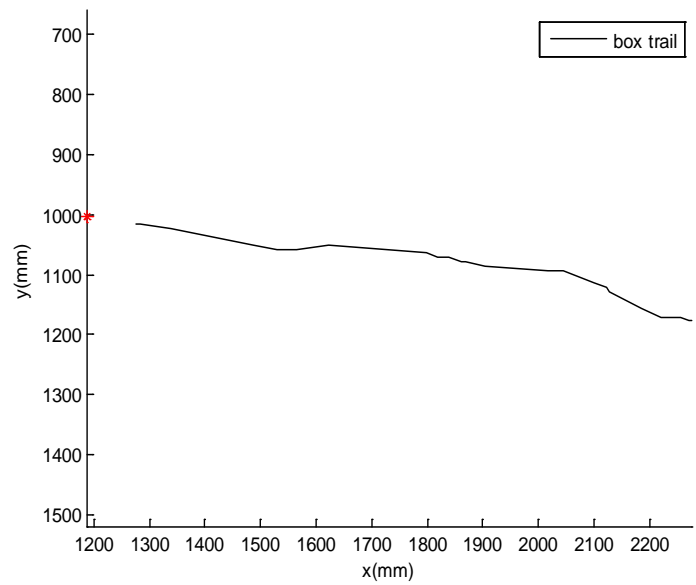

Figure 11. The trajectory of the box 


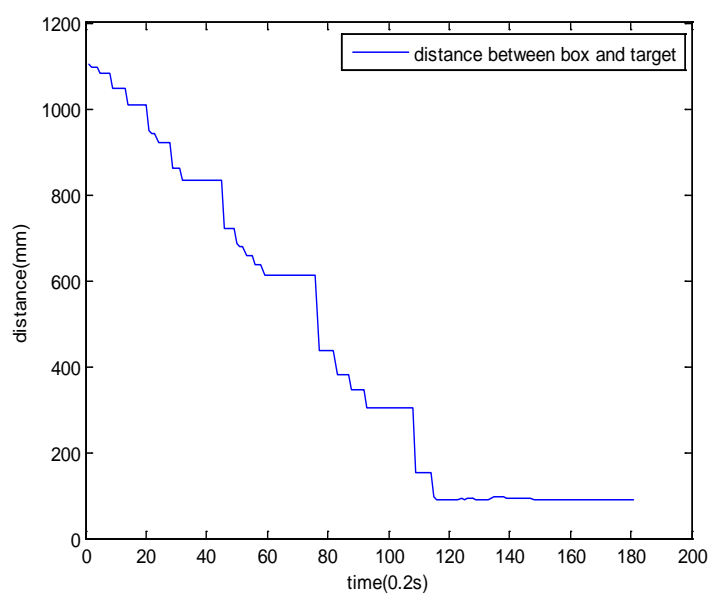

Figure 12. Distance deviation from the box to the Handling goal

\section{Conclusion}

In summary, the controllers were designed in the framework of Leader-Follower mechanism in this paper, in which the leader robot used a LOS PID controller, and a fuzzy controller was adopted by the follower robot. And the amigo bots collaboratively carried the box to the specified destination at last. The proposal was proved by the experiment to be effective.

\section{Acknowledgment}

The research work was supported by Natural Science Foundation of Shandong Province under Grant No. ZR2013FL004, and the Doctoral Foundation of University of Jinan under Grant No. XBS1241.

\section{References}

[1] G. Y. J. D. Parker L E, " Cooperative robot teams applied to the site preparation task," in The 10th International Conference, 2001, pp. 71-77.

[2] E. Tuci, R. Gro, V. Trianni, F. Mondada, M. Bonani, and M. Dorigo, "Cooperation through selfassembly in multi-robot systems," ACM Transactions on Autonomous and Adaptive Systems (TAAS), vol. 1, pp. 115 - 150, 2006.

[3] F. R. Noreils, "Toward a Robot Architecture Integrating Cooperation between Mobile Robots: Application to Indoor Environment," The International Journal of Robotics Research, vol. 12, pp. 79 - 98, 1993.

[4] T. Balch and R. C. Arkin, "Behavior-based formation control for multirobot teams," Robotics and Automation, IEEE Transactions on, vol. 14, pp. 926-939, 1998.

[5] C. B. Low, "A flexible virtual structure formation keeping control design for nonholonomic mobile robots with low-level control systems, with experiments,", 2014, pp. 1576-1582.

[6] S. Yunzhong and Z. Wei, "Leader follower multi-agents network with discrete-time rendezvous via swarm social system strategies,", 2012, pp. 6081-6086.

[7] H. Rezaee, F. Abdollahi and M. B. Menhaj, "Model-free fuzzy leader-follower formation control of fixed wing UAVs,", 2013, pp. 1-5.

[8] P. J. Kennedy and R. L. Kennedy, "Direct versus indirect line of sight (LOS) stabilization," Control Systems Technology, IEEE Transactions on, vol. 11, pp. 3-15, 2003. 
[9] Wu Xiaoping, "Technology research on cooperation base on mutiple AUV,". Shanghai Jiao Tong University, vol. 2008, p. 150.

[10] J. Ghommam, H. Mehrjerdi and M. Saad, "Leader-follower formation control of nonholonomic robots with fuzzy logic based approach for obstacle avoidance,", 2011, pp. 23402345.

[11] B. Li and C. Zhang, "Adaptive fuzzy control for mobile robot obstacle avoidance based on virtual line path tracking,", 2006, pp. 1454-1458. 